\title{
Understanding and Utilizing Patient Preferences in Cancer Treatment Decisions
}

Presented by Peter A. Ubel, MD

\begin{abstract}
Shared decision-making is a complex endeavor that should take into account the patient's personal preferences regarding treatment options. To truly empower patients to be partners in decision-making, especially in situations in which their preferences are important, physicians must learn to communicate better and to distinguish between what is "medical fact" versus a "value judgement." Knowing what are, when to ask, and how to ask the right questions will help physicians be effective in guiding patients toward the right treatments.

J Natl Compr Canc Netw 2016;14(5.5):691-693
\end{abstract}

Patients need clinicians to understand their true preferences for cancer treatment. However, finding out these preferences is a complex challenge, and even the most well-meaning clinicians often miss the mark, according to Peter A. Ubel, MD, Duke-Margolis Center for Health Policy at Duke University, where he is the Madge and Dennis T. McLawhorn University Professor of Business, Public Policy, and Medicine. At the NCCN 21st Annual Conference, Dr. Ubel, a physician and behavioral scientist, used the setting of early-stage prostate cancer to describe the nuances of this endeavor.

"There are clinical situations where you want to engage the patient in decision-making, and the right choice often depends on what the patient cares about. Find out what that is, and you will help the patient get the right treatment," he said.

However, this is easier said than done, Dr. Ubel acknowledged. In a study led by Angie Fagerlin, PhD, Chair of the Department of Population Health Sciences at the University of Utah School of Medicine, Dr. Ubel audiotaped real physician-patient encounters and found that physicians often make treatment recommen-

Presented by Peter A. Ubel, MD, Duke Cancer Institute, The Fuqua School of Business, Durham, North Carolina.

Dr. Ubel has disclosed that he has no financial interests, arrangements, affiliations, or commercial interests with the manufacturers of any products discussed in this article or their competitors.

Correspondence: Peter A. Ubel, MD, Duke Cancer Institute, The Fuqua School of Business, 100 Fuqua Drive, Durham, NC 27708.

E-mail: peter.ubel@duke.edu dations without understanding the patient well enough for these to be "preference-relevant."

"Our research shows there are some real problems in achieving shared decision-making," he said.

\section{Prostate Cancer as an Example}

Dr. Ubel suggested that early-stage prostate cancer is a good example because it has several effective treatment options, including surveillance, surgery, and radiotherapy. Each option has pros and cons, which are weighed differently by different patients.

In brief, Dr. Ubel noted, the conversation with the patient could go something like, "You can have a surveillance strategy that follows up with you every 6 months to see if the cancer is growing to the point where something must be done, or you can have some form of active treatment that eradicates the cancer. What's important is that the different approaches have pros and cons. With active treatment, there's a decent chance you will experience either incontinence or erectile dysfunction. With surveillance, you won't have either of these but you will be living with cancer inside your body, with its accompanying anxiety."

The patient who strongly values maintaining normal erectile function may choose surveillance, whereas the "anxious" patient may choose active treatment (Figure 1). The right choice depends on how patients weigh these trade-offs.

"The question is, how do we figure out which treatment is best for the patient?" he said. 


\section{Preference-Based Recommendation}

Pt: "What would you recommend?"

Dr: "So, surgeons will say surgery...But I think it is really a personal decision that you have to make. Are you the kind of person where the idea of just watching your PSA is that unsettling to you?"

Pt: "Yeah, I think I would."

Dr: "Then I don't think you'd be a good candidate for surveillance."

Figure 1. An example of patient preference-based dialogue for recommendations.

Abbreviation: PSA, prostate-specific antigen.

\section{The Typical Conversation}

Dr. Ubel said that the audiotapes revealed that physicians often give the "bad news," then move hastily to treatment options. A better idea, according to Dr. Ubel, is to assure the patient this is not life-threatening and he "has time" to think, and then have him return in a few weeks to discuss treatment. Educational materials and online decision tools can be useful in the interim.

"My view is that people need time to recover from bad news before making complex decisions," Dr. Ubel said.

According to his observations, physicians discuss treatment alternatives and their risks and benefits a great deal, but spend far less time asking about the patient's understanding of the options and his preferences. Information such as biopsy results and Gleason scores can be hard to absorb, especially in "medicalese," Dr. Ubel pointed out.

"With shared decision-making, you have to make sure patients are informed of the alternatives, but you need to know how to inform them effectively. And regardless of whether it's jargon or not, too much information at one time is hard to comprehend and makes it even harder to make choices."

With too much information in front of him, the patient often pivots to, "You're the doctor, what do you think I should do?"

"You overwhelm them with information and treatment alternatives and they will give up and ask you what to do," he said. "I morally don't recommend that. Instead, give them the right amount of information at the right time so they can be engaged in the choice."
The patient needs time to acknowledge and deal with his emotions and to absorb the information and reflect on it, and the physician needs time to uncover the patient's values.

\section{Giving Advice More Sensitively}

Research has shown that physicians strongly influence their patients' decisions, and although physician recommendations are always appropriate, "I don't think we often do this well," Dr. Ubel said.

Recommendations should be made only after the physician truly understands the patient's value system. And although physicians claim they understand these, Dr. Ubel's observations are that they usually do not. For example, in prostate cancer, physicians are good at evaluating for baseline erectile function (at least through conversation with the patient), but they are not very good at eliciting how patients feel about its importance. In his studies, only about 1 in 10 encounters involved questions that would elicit this information, which is important in guiding treatment selection.

"We don't have evidence that the recommendations made by physicians are following deep discussions," he said. "We poured through these interactions looking for other cues that doctors were picking up on-about what patients cared about-but we didn't find them. Instead, we found patients having to push back, and it took a rare, assertive patient to do this."

When patients ask, "What would you do if you were me?" the physician should seize this opportunity to learn more about that patient. This is the time for the physician to respond, "I may be the expert on the medical facts, but you are the expert on you. I might make different choices than you because I have different preferences. Let me find out more about you."

"This can trigger a productive shared decisionmaking conversation," Dr. Ubel said.

Studies have shown, in fact, that in hypothetical medical situations, physicians typically choose one treatment option for themselves but a different one for their patients. This lends credence to the concept that recommendations are, and should be, individualized.

Finally, clinicians are often biased toward the treatment that aligns with their medical specialty. According to Dr. Ubel, patients often realize this but 
don't care: they express trust in physicians who disclose their personal biases and often follow their recommendations in spite of knowing they are biased.

\section{Teach Back}

Dr. Ubel told attendees that learning the "teach back" strategy will help physicians have more sensitive and productive conversations in a less timeconsuming manner. One should never assume the patient understands the relevant information. Instead, the physician might say, "I've given you lots of information, and I want to make sure I did a good job. Can you tell me in your own words what you heard me saying?" This triggers information that is valuable for continuing the conversation.

"If you use 'teach back' enough, you hone to what your patients need to know, your communication skills become better, and the visits can be more productive and tighter," Dr. Ubel said. Unfortunately, most physicians are not "teaching back," he added. "In our 200 or so taped encounters, we saw no examples of this." 